\section{Towards a further understand- ing of prenatal thyroid theory of homosexuality: Autoimmune thyroiditis, polycystic ovary syndrome, autism and low birth weight}

\section{Osman Sabuncuoglu \\ Department of Child and Adolescent Psychiatry, School of Medicine, Marmara University Hospital, Istanbul, Turkey}

\begin{abstract}
Research into the neurobiological origins of same-sex attraction is inconclusive. A recent theory of homosexuality posited that maternal thyroid dysfunction during pregnancy is associated with an increased rate of homosexual orientation in offspring. Relevant studies from the prenatal thyroid model perspective were reviewed, the major findings of which are as follows: i) An increased prevalence of Hashimoto's disease in lesbian women suggests a maternal and even familial presence of the same autoimmune thyroid disease. Female-tomale transsexuals and lesbian women were also reported to have higher rates of polycystic ovary syndrome (PCOS). Over the last several years, reports suggesting a strong link between PCOS and thyroid autoimmunity have accumulated. ii) The increased risk of autism spectrum disorders (ASD) in the offspring of mothers with thyroid autoimmunity in pregnancy and the association between ASD and gender dysphoria indicate a link between maternal thyroid dysfunction and gender dysphoria/same-sex attraction in the offspring. iii) The high risk of miscarriage and retarded fetal growth in pregnancies of mothers who give birth to homosexual offspring can be explained by the impact of maternal thyroid dysfunction during pregnancy. This perspective review highlights relevant research findings and integrates them into the prenatal thyroid model of homosexuality. A better understanding of the mechanisms involved in the generation of same-sex orientation will contribute to the betterment of individual lives, as well as of society.
\end{abstract}

\section{Introduction}

Although much progress has been made in the scientific understanding of human behavior in the last few decades, the neuro- biological basis of homosexual behavior still remains unknown. Thus, efforts dedicated to health issues specific to gay and lesbian people are produced without the knowledge of the roots of homosexuality. Up until now, several theories ranging from psychosocial ones to biological ones like genetic and prenatal hormonal influences have been put forth to explain the causes of same-sex attraction in human beings, but without reaching definitive conclusions. Recently, as a further effort, a new model that explains homosexual behavior in the offspring as a consequence of suboptimal maternal thyroid production in utero, has emerged. ${ }^{1}$ Because of the increased metabolic demands of pregnancy, the thyroid system is already vulnerable during that time. The most common disorders that affect the thyroid during pregnancy are hyper- and hypothyroidism and their variants, isolated hypo-T4, autoimmune thyroid disease and different types of goitre. ${ }^{2}$ In the paper the thyroid model was proposed, Sabuncuoglu also provided case notes to enable the reader to understand the developmental unfolding of gender nonconformity and same-sex attraction related to maternal thyroid dysfunction in utero. ${ }^{1}$ Diminished supply of thyroid hormones, which are crucial for fetal brain development and programming, androgen decrease secondary to thyroid dysfunction, and neurotoxic effects of maternal autoimmune disease (Hashimoto's thyroiditis in particular) upon fetal brain were suggested as possible mechanisms leading to same-sex orientation in the offspring. ${ }^{1}$

Before the prenatal thyroid model was proposed, thyroid dysfunction had been mentioned in two articles on different aspects of same-sex attraction. In 2005, Ellis and Hellberg 3 reported that fetal exposure to thyroid medications and diet pills was significantly higher in homosexually oriented female offspring. As the study was performed on a non-clinical population, diagnostic data were not available, which made further interpretation difficult. Synthetic thyroid medications, in addition to their use in the treatment of hypothyroidism, are also used for psychiatric indications and even for weight gain. Despite the fact that the study failed to find significance in thyroid medication use in the mothers of homosexual males, the significant result for females must be considered evidence of the role played by thyroid dysfunction during pregnancy.

In a recent study, autoimmune thyroid diseases were mentioned among a group of autoimmune diseases that were found at increased rates in same-sex married Danish individuals. ${ }^{4}$ One of them, Hashimoto's thy-
Correspondence: Osman Sabuncuoglu, Marmara University, School of Medicine, Department of Child and Adolescent Psychiatry, Fevzi Cakmak Mah. Muhsin Yazicioglu Cad. No:10, 34899 Pendik, Istanbul, Turkey.

Tel.: +90.216.6254619 - Fax: +90.216.6254639. E-mail: sabuncuoglu2004@yahoo.com, sabuncuoglu@hotmail.com

Key words: homosexuality, gender dysphoria, autoimmune thyroiditis, polycystic ovary syndrome, autism, birth weight.

Conflict of interest: The author declares no potential conflict of interest.

Received for publication: 21 July 2017. Revision received: 12 September 2017. Accepted for publication: 15 September 2017.

This work is licensed under a Creative Commons Attribution-NonCommercial 4.0 International License (CC BY-NC 4.0).

(C) Copyright O. Sabuncuoglu, 2017

Licensee PAGEPress, Italy

Mental Illness 2017; 9:7325

doi:10.4081/mi.2017.7325

roiditis, warrants particular attention. Hashimoto's disease is characterized by the production of immune cells and autoantibodies that attack the thyroid tissue, which is followed by the gradual destruction and hypofunction of the gland.5 Hashimoto's thyroiditis is one of the most prevalent autoimmune diseases, estimated to affect more than $10 \%$ of the population, and is seven times more likely to be seen in females than in males. Having a strong genetic basis, Hashimoto's disease tends to run in families, and most clinicians view it as a mother-daughter disease. Thus, there is sufficient ground to assert that the significance of female Hashimoto's cases in the Frisch et al. study ${ }^{4}$ may suggest for the presence of autoimmune thyroid disease in their mothers. In Sabuncuoglu's sample, ${ }^{1}$ the two female adolescents whose mothers had Hashimoto's thyroiditis also had Hashimoto's thyroiditis themselves. Apparently, male offspring affected by maternal thyroid dysfunction during pregnancy, in terms of same-sex attraction, did not surface in the Danish study, as they were less likely to develop Hashimoto's thyroiditis themselves. Another point requiring attention is that the prevalence of females with Hashimoto's disease in the Danish register was $0.04 \%$, which is far behind the general population estimates of $10 \%$. A much higher number of cases are expected to occur according to population 
projections. The prenatal thyroid model of homosexuality is in its infancy and there is much to be done to advance its development. In this perspective paper, the aim was to review the relevant studies from the prenatal thyroid theory standpoint, which has been overlooked to date.

\section{Polycystic ovary syndrome in lesbian women/female-to-male transsexuals and autoimmune thyroiditis}

Polycystic ovary syndrome (PCOS) is a common endocrine disorder that affects up to $10 \%$ of women of reproductive age. PCOS diagnosis requires the presence of two of the following three criteria: 1. polycystic ovaries on pelvic ultrasound examination, 2. clinical features or biochemical features of hyperandrogenism and 3. oligoovulation or anovulation. ${ }^{6}$ Although PCOS is recognized as a familial condition, the genes that are involved in the etiology of the syndrome have not been well-established, nor have the environmental factors that contribute to their expression. ${ }^{7}$ Although PCOS is one of the leading causes of infertility, many women with PCOS get pregnant and deliver healthy babies, either naturally or with medical intervention (subfertility). Studies have revealed an increased prevalence of female-to-male transsexuals and lesbian women with PCOS. As far as we know, the earliest study was conducted by Futterweit et al., ${ }^{8}$ who found an increased rate of PCOS in female-to-male transsexuals. Then, Balen et al. ${ }^{9}$ demonstrated 93\% pelvic ultrasound diagnosis of polycystic ovaries in a sample of 16 female-to-male transsexuals who had no history of exogenous androgen therapy. This was followed by an article on hyperandrogenic disorders in female-to-male transsexuals which concluded that although a higher prevalence of PCOS (50\% versus 5\% in the female population) was evident, the mechanism behind that phenomenon remained unknown. ${ }^{10}$ Finally, a recent study from Japan, which investigated 69 cases, confirmed the finding that female-to-male transsexuals were more likely to have PCOS (58\%) and hyperandrogenaemia. ${ }^{11}$ Likewise, in a systematic study of consecutive women attending a fertility clinic, Agrawal et al. ${ }^{6}$ found that selfidentified lesbian women were significantly more likely to have PCOS than heterosexual women $(38 \%$ versus $14 \%, \mathrm{P}<0.001)$. Thus, it appears that the finding of PCOS in female-to-male transsexuals is consistent in the medical literature, and there is also evidence for lesbian women. So, then, what is the relevance of those PCOS research to the prenatal thyroid theory of homosexuality? Or isn't it even a counter-argument against the prenatal thyroid theory? The answer is as follows.

Over the last decade, there is growing interest among researchers in the investigation of the association between PCOS and thyroid autoimmunity. In the first systematic prospective study addressing this issue, significantly elevated levels of thyroid peroxidase (TPO) or thyroglobulin (Tg) antibodies distinctive for Hashimoto's thyroiditis were found in $26.9 \%$ of 175 PCOS patients, in contrast to $8.3 \%$ of 168 controls. ${ }^{12}$ A meta-analysis of 6 studies published until 2013 concluded that the levels of serum thyroid-stimulating hormone (TSH), anti-TPO and anti-Tg rates were all significantly higher in a total of 726 PCOS patients than in 879 controls, suggesting that PCOS may be a kind of autoimmune disease in close association with autoimmune thyroiditis. ${ }^{13}$ Afterwards, similar studies confirming that association were published as well. ${ }^{14,15}$ The increased prevalence of PCOS was also demonstrated in 172 girls with chronic lymphocytic thyroiditis when compared to controls (46.8 versus $4.3 \%, \mathrm{P}=0.001) .{ }^{16}$ At present, there is agreement that PCOS and thyroid autoimmunity are interrelated and often coexist in the same patient. However, the pathophysiological mechanism and contributing factors behind this association are unknown, and this requires further research.

All of these data indicate that if PCOS prevalence is higher in women with samesex attraction, then thyroid autoimmunity would also be expected to increase in the same group, in contrast to population estimates. In the present article, for the first time, the significance of this comorbidity for homosexual females has been highlighted. It is apparent that, the thyroid factor was present alongside PCOS, and we hereby reach a further and deeper understanding of homosexuality in females. Figure 1 provides a schematic representation of the prenatal thyroid model along with lifetime influences in females.

\section{Gender dysphoria in autism spectrum disorder and maternal thyroid dysfunction}

Autism spectrum disorders (ASD) are a group of neurodevelopmental disorders characterized by persistent deficits in social interaction and communication, and repetitive and stereotyped patterns of behavior, often accompanied by intellectual impairment. ${ }^{17}$ Despite the fact that various genetic and environmental factors, along with significant findings, have been implicated in the etiology of ASD, none of them is conclusive and research still persists. A possible link between hypothyroidism in infancy and autism was first mentioned by Gillberg et $a l .18$ In the earliest study that could be located, Sweeten et al. ${ }^{19}$ presented evidence that hypothyroidism/Hashimoto's thyroiditis was more common in 101 families with ASD probands than in the healthy control families $\left(X^{2}=13.21 ; \mathrm{P}=0.0003\right)$. The authors suggested, as a possible explanation, an autoimmune process of the mothers that is targeting the developing fetus in utero. 19 Further, in a multicenter study of 308 children with ASD, researchers identified autoimmune thyroid disorder as a risk factor for autistic regression (adjusted odds ratio $(\mathrm{OR})=2.09 ; 95 \%$ confidence interval [CI]: $1.28,3.41) .{ }^{20}$ In addition, the TPO gene was found to be associated with ASD after a multiple-comparisons correction. ${ }^{21}$ In 2013, results from the Generation $\mathrm{R}$ Study, which was a mother-child cohort study, revealed that severe maternal hypothyroxinemia $(n=136)$ increased the odds of having a child with autism almost 4fold (adjusted OR=3.89, 95\% CI: 1.83 , 8.20, $\mathrm{P}<0.001) .{ }^{22}$ The authors concluded that disruption of the reelin-dab signaling system resulting from early maternal hypothyroxinemia may lead to the development of the neuropathological abnormalities characteristic of autism. A Danish population-based cohort study yielded similar results. ${ }^{23}$ Recently, it was reported that, even if measures of maternal thyroid hormones were within the normal range, the presence of TPO antibodies significantly predicted the risk of ASD in the offspring $(\mathrm{OR}=1.78,95 \% \mathrm{CI}: 1.16,2.75$, $\mathrm{P}=0.009) .24$ The authors pointed out the possibility that maternal TPO antibodies may even serve as a predictive marker for the risk of ASD in the child. In summary, the results of a meta-analysis and review comprising 9775 cases indicated that maternal autoimmune disease, and more specifically autoimmune thyroid disease, appeared to be an independent risk factor of ASD in the offspring. ${ }^{25}$

A growing number of reports indicate that ASDs are overrepresented among minors with gender dysphoria. In the DSM5,17 the term gender dysphoria is defined as distress resulting from marked incongruence between one's experienced/expressed gender and his or her assigned gender, together with a persistent and strong desire to be of another gender. As most transsexuals engage in homosexual behavior, the study of gender dysphoria may provide data relevant to the origins of same-sex orienta- 
tion. The existing literature regarding the ASD-gender dysphoria link consists of mostly case reports and a few observational studies. A recent review of the robust studies conducted in this area concluded that the prevalence of ASD in children and adolescents with symptoms of gender dysphoria were considerably higher than in the general population. 26 According to another review, ${ }^{27}$ as many as $20 \%$ of individuals attending gender identity clinics may show some signs of ASD; there is a frequent cooccurrence of both conditions. Undoubtedly, the impact of ASD on normal development is pervasive, including in gender-specific behavior, and there are conceptual challenges to understanding gender dysphoria in terms of comorbidity. 28 The authors of the aforementioned reviews are in agreement that the etiological mechanism of the co-occurrence of gender dysphoria and ASD is presently unknown.

Interestingly, a recent study reported that children born to women with PCOS have a higher risk of developing ASD.29 Given that PCOS and thyroid autoimmunity are highly comorbid, it is not surprising to find similar results in different studies. As noted in the upper section, consequences of this comorbidity are also expected to involve the issues relating to sexual orientation.

Once again, the time is ripe to direct attention to the effects of maternal thyroid autoimmunity and hypothyroidism on the offspring. As research has demonstrated, if maternal thyroid autoimmunity is associated with ASD in the offspring, who are more likely to display gender dysphoria, there is the possibility of an association between maternal thyroid autoimmunity and gender dysphoria. Figure 2 shows the indirect research evidence along with direct evidence, which is the prenatal thyroid model. ${ }^{1}$ Although the focus of Sabuncuoglu's study was on gender nonconformity/homosexuality, ${ }^{1}$ it was suggested that gender dysphoria be considered among the consequences of prenatal thyroid dysfunction in the offspring. Further research may provide accurate findings regarding these issues.

It must be noted that the aforementioned findings cannot be applied to all individuals with ASD or gender dysphoria. The timing, duration, severity and type of insult during development determine the specific consequence for an individual. ${ }^{30} \mathrm{We}$ also recommend against stigmatization of patients based on the associations presented here.

\section{Higher risk of miscarriage and retarded fetal growth in mothers of homosexual offspring and maternal thyroid dysfunction}

Many studies have investigated associations among birth weight, sexual orientation and sibship status, ${ }^{31-33}$ and some mildly significant results for males have been reported. It is hypothesized that the maternal immune reaction against the male fetus may adversely affect specific brain areas associated with typical sexual orientation, and this effect may correlate with the degree of fetal growth retardation. ${ }^{31-33}$ In a recent study, a distinct type of maternal immune response implicated in the etiology of male sexual orientation was investigated, with low birth weight and high fetal loss used as markers. ${ }^{34}$

Like those of previous indirect measures of research used in the study of sexual orientation, low birth weight and high fetal loss are neither specific nor sensitive enough to be employed as markers. There are many contributing factors associated with low birth weight and its consequences. ${ }^{35}$ The same also applies to fetal loss and miscarriage. ${ }^{36}$ The United Nations Children's Fund and World Health Organization do not recommend the use of low birth weight as a proxy for any single dimension of either maternal or perinatal health outcomes. ${ }^{37}$ It is necessary to take into account all factors that may be related if non-specific measures are used to test a certain objective.

Thyroid disorders are prevalent in women of childbearing age and tend to become more prevalent with age. ${ }^{38}$ There is agreement that significant associations exist between thyroid disorders and abnormalities of the reproductive system, both in males and in females. ${ }^{38}$ Besides resulting in infertility/subfertility, thyroid dysfunction can also impact fetal development and even neonatal life. Among other adverse outcomes, increased rate of miscarriage and low birth weight are well established. ${ }^{38}$

Despite high prevalence of thyroid disorders in females, male offspring are more likely to be affected by thyroid dysfunction in utero. Congenital hypothyroidism $(\mathrm{CH})$ is a condition characterized by a deficiency or lack of thyroid hormone that occurs in a newborn. $\mathrm{CH}$ is further classified as either permanent or transient. Permanent congenital hypothyroidism $(\mathrm{PCH})$ is seen mostly as

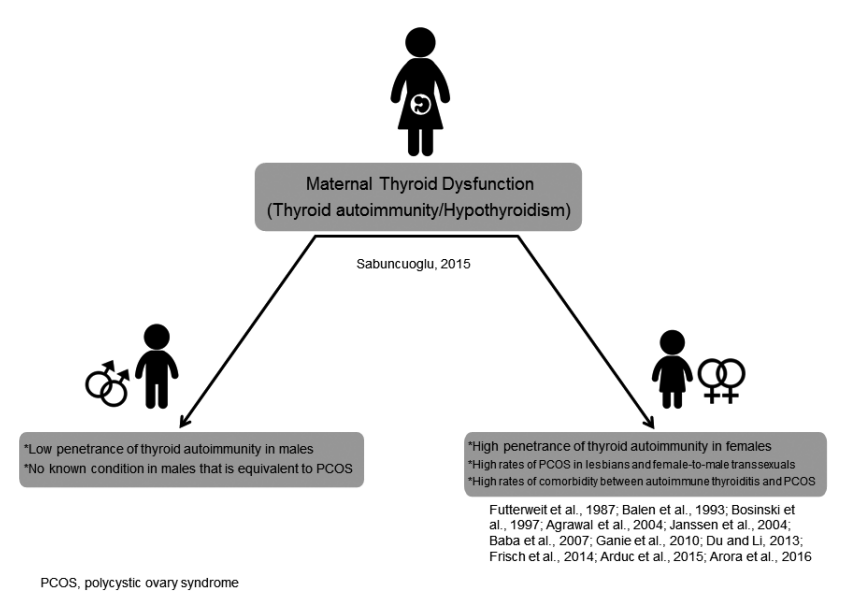

Figure 1. Schematic representation of the mechanisms involved in the prenatal thyroid model of homosexuality with lifetime influences in females.

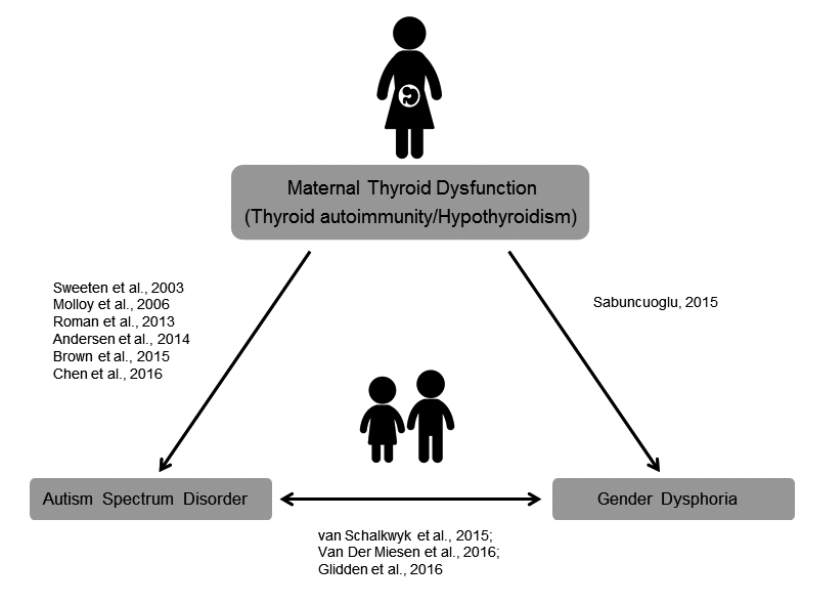

Figure 2. Direct and indirect evidences of association between maternal thyroid dysfunction and autism spectrum disorder/gender dysphoria in the offspring. 
a result of thyroid gland dysgenesis $(85 \%)$ and inborn errors of thyroid hormone biosynthesis and requires a lifetime thyroid hormone replacement. ${ }^{39}$ In transient congenital hypothyroidism (TCH), extrinsic factors, such as in utero exposure to maternal thyrotropin receptor-blocking antibodies, exposure to antithyroid drugs, iodine deficiency and iodine excess, are responsible, and thyroid hormone replacement is necessary only in infancy. 39 Only a small proportion of infants born to mothers with thyroid dysfunction develop TCH. Females are twice as likely to be affected by $\mathrm{PCH}$, while researchers from Iran, Turkey and Italy noted that female/male ratios range from 0.43 to 0.70 for TCH. ${ }^{40-42}$ In addition, the infants with TCH had lower birth weight than infants with PCH.40-42 Thus, the contrast between $\mathrm{PCH}$ and $\mathrm{TCH}$ provides a unique opportunity for the study of the effect of maternal thyroid dysfunction on the sexual orientation of the offspring. Increased male preponderance and low birth weight are significant parallels between TCH and aforementioned studies of homosexuality. However, no research data are available regarding an association between any form of $\mathrm{CH}$ and sexual orientation. Sabuncuoglu reported only one boy with gender nonconformity who had a history of TCH along with a history of maternal thyroid disease. ${ }^{1}$ This suggests that individuals born to mothers with thyroid dysfunction during pregnancy may develop gender nonconformity/same-sex attraction without having signs and symptoms of TCH in infancy. Most infants with $\mathrm{CH}$ appear normal at birth and show no signs of $\mathrm{CH} .43$ We should note that although Hashimoto's thyroiditis was not mentioned among the causes of TCH, Ozdemir et al. drew attention to the role of Hashimoto's thyroiditis in the pathogenesis of TCH. 44

In summary, it is apparent that the significant findings of low birth weight and high fetal loss in the aforementioned studies are consistent with what the prenatal thyroid theory of homosexuality states. ${ }^{1}$

\section{Discussion and Conclusions}

In this perspective paper, study findings from relevant papers were highlighted and mapped onto the theoretical framework of the prenatal thyroid model of homosexuality. We believe that a more advanced and deeper understanding of the theory has been achieved with this work.

Homosexuality is not considered a behavioral disorder since the release of DSM-III, ${ }^{45}$ and this approach should be maintained without compromise. It appears that homosexuality is not a disease in itself but rather the result of a number of medical issues, with thyroid dysfunction being the most prominent one. As it emerges that there is such a complex topic, which may be conceptualized in terms of reproductive health but is not limited to that, a multidisciplinary approach and professional awareness are required.

It should be emphasized that the thyroid dysfunctions in mothers noted as goitre and hypothyroidism in Sabuncuoglu's study 1 may in fact represent Hashimoto's thyroiditis, as they are well-established and even common outcomes of autoimmune thyroiditis. The Istanbul region, from which Sabuncuoglu's study population was drawn, ${ }^{1}$ has been reported to be an iodinesufficient area. ${ }^{46}$ So, it is unlikely that the maternal thyroid problems reported as hypothyroidism and goitre were due to iodine insufficiency.

It turns out that, in addition to maternal thyroid autoimmunity affecting the fetus in utero, female sexual orientation is also affected by an individual's own autoimmune thyroid disease, which is inherited and unfolds during the course of development. Butch and femme sex roles among lesbians refer to whether the predominant sexual identity and behavior is masculine (butch) or feminine (femme). ${ }^{47}$ It may be that the in utero influence of maternal thyroid dysfunction rather leads to the development of femme-type lesbian behavior, and in utero influence together with later-onset PCOS may cause the development of butchtype behavior. Increased rates of PCOS and presumed thyroid autoimmunity in femaleto-male transsexuals, which could be considered the high-end of female homosexuality, reveals insight regarding the mechanism of the entire spectrum of female homosexuality.

Since there is an increased comorbidity between thyroid autoimmunity and PCOS, another question is whether comorbid PCOS in maternal thyroid dysfunction may affect the fetus in utero. Further research may shed light on this issue.

It has also been shown that Hashimoto's thyroiditis is frequently associated with non-thyroidal autoimmune diseases, with arthropathies and connective tissue diseases being the most common ones. 48 So can there be a kind of parallel autoimmune mechanism masked by thyroid dysfunction that leads to homosexual behavior in the offspring? As of now, the answer to this is not known. Presently, there is one bird in hand and evidence for others needs to be demonstrated.

There are certain difficulties in the diagnosis of thyroid disorders. US Census data suggest that approximately 15 million adults (almost half of the total) have unrecognized thyroid disease, including subclinical hypothyroidism. ${ }^{49}$ The same is true for the European population that a large proportion unknowingly has laboratory evidence of thyroid dysfunction. 50 It has only been within the last couple of decades that common thyroid antibody tests became widely available, which are crucial for the diagnosis of autoimmune thyroid dysfunction. Thus, the more we learn about the thyroid gland and its disorders, the more we will understand the precise mechanisms affecting sexual orientation. A comprehensive consideration of all thyroid issues is needed in the study of gender nonconformity and same-sex attraction.

Since ancient times, mankind has searched for an explanation of same-sex attraction. Long ago, the mission of medicine was described by Avicenna as follows: ...To know fully about each of these (how health is maintained and how health is lost) we must ascertain the causes of both health and sickness. ${ }^{51}$ Medicine has been pursuing this mission for centuries. The present work should be viewed as another addition to all the efforts undertaken in this field. We conclude that time is running out, and the mystery surrounding the origins of same-sex attraction may be solved at last. Further steps are needed so that medical science can make a positive difference in individual lives, as well as in society.

\section{References}

1. Sabuncuoglu O. High rates of same-sex attraction/gender nonconformity in the offspring of mothers with thyroid dysfunction during pregnancy: proposal of prenatal thyroid model. Ment Illn 2015;7:5810.

2. Krassas G, Karras SN, Pontikides N. Thyroid diseases during pregnancy: a number of important issues. Hormones (Athens) 2015;14:59-69.

3. Ellis L, Hellberg J. Fetal exposure to prescription drugs and adult sexual orientation. Pers Individ Dif 2005;38:22536.

4. Frisch M, Nielsen NM, Pedersen BV. Samesex marriage, autoimmune thyroid gland dysfunction and other autoimmune diseases in Denmark 1989-2008. Eur J Epidemiol 2014;29:63-71.

5. Zaletel K, Gaberšček S. Hashimoto's thyroiditis: from genes to the disease. Curr Genomics 2011;12:576-88.

6. Agrawal R, Sharma S, Bekir J, et al. Prevalence of polycystic ovaries and polycystic ovary syndrome in lesbian women compared with heterosexual women. Fertil Steril 2004;82:1352-7. 
7. Prapas N, Karkanaki A, Prapas I, et al. Genetics of polycystic ovary syndrome. Hippokratia 2009;13:216-23.

8. Futterweit W, Weiss RA, Fagerstrom RM. Endocrine evaluation of forty female-to-male transsexuals: increased frequency of polycystic ovarian disease in female transsexualism. Arch Sex Behav 1986;15:69-78.

9. Balen AH, Schochter ME, Montgomery $\mathrm{D}$, et al. Polycystic ovaries are a common finding in untreated female to male transsexuals. Clin Endocrinol 1993;38:325-9.

10. Bosinski HA, Peter M, Bonatz G, et al. A higher rate of hyperandrogenic disorders in female-tomale transsexuals. Psychoneuroendocrinology 1997;22: 361-80.

11. Baba T, Endo T, Honnma H, et al. Association between polycystic ovary syndrome and female-to-male transsexuality. Hum Reprod 2007;22:1011-6.

12. Janssen OE, Mehlmauer N, Hahn S, et al. High prevalence of autoimmune thyroiditis in patients with polycystic ovary syndrome. Eur J Endocrinol 2004;150: 363-9.

13. Du D, Li X. The relationship between thyroiditis and polycystic ovary syndrome: a meta-analysis. Int J Clin Exp Med 2013;6:880-9.

14. Arduc A, Aycicek Dogan B, Bilmez S, et al. High prevalence of Hashimoto's thyroiditis in patients with polycystic ovary syndrome: does the imbalance between estradiol and progesterone play a role? Endocr Res 2015;40:204-10.

15. Arora S, Sinha K, Kolte S, Mandal A. Endocrinal and autoimmune linkage: evidences from a controlled study of subjects with polycystic ovarian syndrome. J Hum Reprod Sci 2016;9:1822.

16. Ganie MA, Marwaha RK, Aggarwal R, Singh S. High prevalence of polycystic ovary syndrome characteristics in girls with euthyroid chronic lymphocytic thyroiditis: a case-control study. Eur J Endocrinol 2010;162:1117-22.

17. American Psychiatric Association. Diagnostic and statistical manual of mental disorders, (DSM-5®). Washington, DC: American Psychiatric Pub; 2013.

18. Gillberg IC, Gillberg C, Kopp S. Hypothyroidism and autism spectrum disorders. J Child Psychol Psychiatry 1992;33:531-42.

19. Sweeten TL, Bowyer SL, Posey DJ, et al. Increased prevalence of familial autoimmunity in probands with pervasive developmental disorders. Pediatrics 2003;112:e420.
20. Molloy CA, Morrow AL, Meinzen-Derr J, et al. Familial autoimmune thyroid disease as a risk factor for regression in children with Autism Spectrum Disorder: a CPEA Study. J Autism Dev Disord 2006;36:317-24.

21. Ramos PS, Sajuthi S, Langefeld CD, Walker SJ. Immune function genes CD99L2, JARID2 and TPO show association with autism spectrum disorder. Mol Autism 2012;3:4.

22. Román GC, Ghassabian A, BongersSchokking JJ, et al. Association of gestational maternal hypothyroxinemia and increased autism risk. Ann Neurol 2013;74:733-42.

23. Andersen SL, Laurberg P, Wu CS, Olsen J. Attention deficit hyperactivity disorder and autism spectrum disorder in children born to mothers with thyroid dysfunction: a Danish nationwide cohort study. BJOG 2014;121:1365-74.

24. Brown AS, Surcel HM, Hinkka-YliSalomäki S, et al. Maternal thyroid autoantibody and elevated risk of autism in a national birth cohort. Prog Neuropsychopharmacol Biol Psychiatry 2015;57:86-92.

25. Chen SW, Zhong XS, Jiang LN, et al. Maternal autoimmune diseases and the risk of autism spectrum disorders in offspring: a systematic review and metaanalysis. Behav Brain Res 2016;296:61-9.

26. Glidden D, Bouman WP, Jones BA, Arcelus J. Gender dysphoria and autism spectrum disorder: a systematic review of the literature. Sex Med Rev 2016;4:3-14.

27. Van Der Miesen AI, Hurley H, De Vries AL. Gender dysphoria and autism spectrum disorder: A narrative review. Int Rev Psychiatry 2016;28:70-80.

28. van Schalkwyk GI, Klingensmith K, Volkmar FR. Gender identity and autism spectrum disorders. Yale J Biol Med 2015;88:81-3.

29. Kosidou K, Dalman C, Widman L, et al. Maternal polycystic ovary syndrome and the risk of autism spectrum disorders in the offspring: a populationbased nationwide study in Sweden. Mol Psychiatry 2016;21:1441-8.

30. Fowden AL, Giussani DA, Forhead AJ. Intrauterine programming of physiological systems: causes and consequences. Physiology (Bethesda) 2006;21:29-37.

31. Blanchard R, Ellis L. Birth weight, sexual orientation and the sex of preceding siblings. J Biosoc Sci 2001;33:451-67.

32. Blanchard R, Zucker KJ, Cavacas A, et al. Fraternal birth order and birth weight in probably prehomosexual feminine boys. Horm Behav 2002;41:321-7.
33. VanderLaan DP, Blanchard R, Wood H, et al. Birth weight and two possible types of maternal effects on male sexual orientation: a clinical study of children and adolescents referred to a Gender Identity Service. Dev Psychobiol 2015;57:25-34.

34. Skorska MN, Blanchard R, VanderLaan DP, et al. Gay male only-children: Evidence for low birth weight and high maternal miscarriage rates. Arch Sex Behav 2017;46:205-15.

35. Pschirrer ER, Little GA. Fetal assessment. In: McInerny TK, Adam HM, Campbell DE, et al, eds. American Academy of Pediatrics Textbook of Pediatric Care. Elk Grove Village, IL: American Academy of Pediatrics; 2009.

36. Regan L, Backos M, Farquharson RG. Recurrent miscarriage. In: James DK, Steer PJ, Weiner CP, Gonik B, eds. High Risk Pregnancy: Management Options. 4th ed. St. Louis, MO: W. B. Saunders/Elsevier; 2011.

37. UNICEF and WHO. Low birth weight: Country, regional and global estimates. New York: UNICEF; 2004.

38. Krassas GE, Poppe K, Glinoer D. Thyroid function and human reproductive health. Endocr Rev 2010;31:70255 .

39. Rastogi MV, LaFranchi SH. Congenital hypothyroidism. Orphanet J Rare Dis 2010;5:17.

40. Ghasemi M, Hashemipour M, Hovsepian S, et al. Prevalence of transient congenital hypothyroidism in central part of Iran. J Res Med Sci 2013;18:699-703.

41. Kara C, Günindi F, Can Yılmaz G, Aydin M. Transient congenital hypothyroidism in Turkey: An analysis on frequency and natural course. J Clin Res Pediatr Endocrinol 2016;8:170-9.

42. Oliveri A, Fazzini C, Grandolfo ME, et al. Ipotiroidismo congenito transitorio in aree iodocarenti. Annali dell'Istituto Superiore di Sanità 1998;34;331-6.

43. Büyükgebiz A. Newborn screening for congenital hypothyroidism. J Clin Res Pediatr Endocrinol 2013;5:8-12.

44. Ozdemir H, Akman I, Coskun S, et al. Maternal thyroid dysfunction and neonatal thyroid problems. Int $\mathrm{J}$ Endocrinol 2013;2013:987843.

45. American Psychiatric Association. Diagnostic and statistical manual of mental disorders. 3rd ed. Arlington, VA: Author; 1980.

46. Erdoğan MF, Ağbaht K, Altunsu T, et al. Current iodine status in Turkey. J Endocrinol Invest 2009;32:617-22.

47. Brown WM, Finn CJ, Cooke BM, Breedlove SM. Differences in finger 
length ratios between self-identified butch and femme lesbians. Arch Sex Behav 2002;31:123-7.

48. Ruggeri RM, Trimarchi F, Giuffrida G, et al. Autoimmune comorbidities in Hashimoto's thyroiditis: different patterns of association in adulthood and childhood/adolescence. Eur J
Endocrinol 2017;176:133-41.

49. Cooper DS, Ridgway EC. Thoughts on prevention of thyroid disease in the United States. Thyroid 2002;12:925-9.

50. Garmendia Madariaga A, Santos Palacios S, Guillén-Grima F, Galofré JC. The incidence and prevalence of thyroid dysfunction in Europe: a meta- analysis. J Clin Endocrinol Metab 2014;99:923-31.

51. Gruner OC. A treatise on the canon of medicine of Avicenna. London: Luzac and Co; 1930. Available from: https://archive.org/stream/AvicennasCa nonOfMedicine/9670940-Canon-ofMedicine_djvu.txt 